\title{
Differential expression of leukocyte $\beta 2$ integrin signal transduction-associated genes in patients with symptomatic pulmonary embolism
}

\author{
JIN YUN ${ }^{1}$, QIANGLIN DUAN ${ }^{1}$, LEMIN WANG $^{1}$, WEI LV ${ }^{1}$, ZHU GONG $^{1}$, FAN YANG $^{2}$ and YANLI SONG ${ }^{1}$ \\ Departments of ${ }^{1}$ Cardiology and ${ }^{2}$ Laboratory Medicine, Tongji Hospital, Tongji University, Shanghai 200065, P.R. China
}

Received March 24, 2013; Accepted November 4, 2013

DOI: $10.3892 / \mathrm{mmr} .2013 .1780$

\begin{abstract}
Whole human genome oligo microarrays were employed to systematically investigate the differential expression characteristics of associated mRNAs, which were found in the signal transduction pathway of $\beta 2$ integrins in peripheral blood mononuclear cells (PBMCs) between patients with symptomatic pulmonary embolism (PE) and controls. A total of 20 cases of PE patients and twenty gender- and age-matched controls were recruited for the study. Human cDNA microarray analysis was used to detect the differences in mRNA expression between the two groups and a random variance model corrected t-test was used to analyze the statistical data. A total of 80 associated mRNAs were detected. The mRNA expression of chemokines, ligands, inside-out and outside-in signaling pathway-associated proteins were upregulated significantly in the PE group, compared with the controls. In five subunit-associated mRNAs, the mRNA expression of ITGAL, ITGAM, ITGAX and ITGB2, which encode for the subunits of $\alpha \mathrm{L}, \alpha \mathrm{M}, \alpha \mathrm{X}$ and $\beta 2$, were upregulated in the PE group and the differences, with the exception of ITGB2, were statistically significant $(\mathrm{P}<0.05)$. The mRNA expression of ITGAD was downregulated; however, there was no significant difference $(\mathrm{P}>0.05)$. The expression of $\mathrm{Fgr}$ mRNA was significantly downregulated $(\mathrm{P}<0.01)$. Thus, in PE patients, bilateral signal transduction pathways of $\beta 2$ integrins in neutrophils and monocytes were activated, enhancing innate immunity.
\end{abstract}

\section{Introduction}

Pulmonary embolism (PE), together with deep venous thrombosis (DVT), is termed venous thromboembolism (VTE). Acute venous thrombosis is a type of red thrombus. A large

Correspondence to: Professor Lemin Wang, Department of Cardiology, Tongji Hospital, Tongji University, 389 Xincun Road, Shanghai 200065, P.R. China

E-mail: wanglemin@tongji.edu.cn

Key words: $\beta 2$ integrin, signal transduction, neutrophils, monocytes number of cells gather in red thrombus irregularly and the majority are neutrophils. Smeeth et al observed that VTE was associated with infection, and the risks of DVT and PE were significantly raised in the first two weeks of diagnosis (1). The current study observed that compromised immunity is associated with the occurrence of VTE (2). In 2009, it was reported (3) that the mRNA expression of natural killer (NK) cells and $\mathrm{T}$ lymphocytes in PE patients were significantly downregulated. It has recently been reported that the immunity of $\mathrm{CD}^{+}$and $\mathrm{CD}^{+} \mathrm{T}$ cells in patients with acute $\mathrm{PE}$ were reduced (1) and the immunity of $\mathrm{CD}^{+}$and $\mathrm{CD} 8^{+} \mathrm{T}$ cells in patients with chronic thromboembolic pulmonary hypertension were also reduced (2). These observations indicate that integrins are the core proteins in VTE and $\beta 2$ integrins, which are distributed in the leukocyte and are involved in the occurrence of VTE.

Integrins are type I transmembrane glycoproteins. In humans, there are 24 integrins formed by specific non-covalent associations of $18 \alpha$ and $8 \beta$ subunits (4). A type of integrin may be distributed in a number of cells and a number of integrins are expressed in signal cells.

Numerous integrins are specially expressed on specific types of cells. For example, $\beta 2$ integrins are only expressed on the cytomembrane of leukocytes, thus, $\beta 2$ integrins are also known as leukocyte integrins.

A recent study suggested that the associated mRNA expression of integrins, which were distributed in leukocytes and platelets, were upregulated significantly (5). It is unclear how the integrin-mediated signal transduction pathway and signaling proteins function. To investigate the differential expression of associated mRNAs, which were found in the signal transduction pathway of $\beta 2$ integrins in PE patients, the whole human genome oligo microarray was employed to systematically investigate the expression differences of associated mRNAs.

\section{Materials and methods}

Patient information. A total of 20 patients were enrolled in the PE group and were admitted to Tongji Hospital (Shanghai, China) during 2007, including 11 males and 9 females, with an average age of $70 \pm 14$ years (44-89 years old). All patients were diagnosed with $\mathrm{PE}$ on the basis of a minimum of two of 
Table I. mRNA expression of genes related to the subunits of $\beta 2$ integrins.

\begin{tabular}{|c|c|c|c|c|c|}
\hline Subunit & Gene & PE & Control & P-value & P-value \\
\hline$\beta 2$ & ITGB2 & $17.9 \pm 0.40$ & $17.88 \pm 0.29$ & - & 0.834460 \\
\hline$\alpha \mathrm{L}$ & ITGAL & $14.00 \pm 0.64$ & $13.51 \pm 0.67$ & $<0.05$ & 0.024773 \\
\hline$\alpha \mathrm{M}$ & ITGAM & $16.89 \pm 0.60$ & $16.34 \pm 0.31$ & $<0.01$ & 0.000830 \\
\hline$\alpha X$ & ITGAX & $13.91 \pm 0.60$ & $12.84 \pm 0.43$ & $<0.01$ & 0.001798 \\
\hline$\alpha \mathrm{D}$ & ITGAD & $9.118 \pm 0.82$ & $9.38 \pm 1.13$ & - & 0.446025 \\
\hline
\end{tabular}

P-values denote PE vs. control groups. PE, pulmonary embolism.

Table II. mRNA expression of genes related to the ligand of $\beta 2$ integrins.

\begin{tabular}{|c|c|c|c|c|c|}
\hline Ligand & Gene & $\mathrm{PE}$ & Control & P-value & P-value \\
\hline ICAM-1 & ICAM1 & $13.45 \pm 0.82$ & $12.88 \pm 0.48$ & $<0.05$ & 0.01456 \\
\hline ICAM-2 & ICAM2 & $15.13 \pm 0.60$ & $15.42 \pm 0.36$ & - & 0.07313 \\
\hline ICAM-3 & ICAM3 & $17.84 \pm 0.43$ & $17.43 \pm 0.37$ & $<0.01$ & 0.00253 \\
\hline ICAM-4 & ICAM4 & $9.18 \pm 0.51$ & $9.09 \pm 0.61$ & - & 0.61033 \\
\hline ICAM-5 & ICAM5 & $7.78 \pm 0.59$ & $7.60 \pm 0.77$ & - & 0.30345 \\
\hline JAM-1 & F11R & $15.64 \pm 1.04$ & $15.5 \pm 0.56$ & - & 0.45364 \\
\hline JAM-3 & JAM3 & $9.9 \pm 0.88$ & $9.47 \pm 0.74$ & $<0.05$ & 0.03685 \\
\hline RAGE & AGER & $14.90 \pm 0.37$ & $14.37 \pm 0.28$ & $<0.01$ & $1.1 \mathrm{E}-05$ \\
\hline \multirow[t]{3}{*}{ Fibrinogen } & FGA & $4.65 \pm 0.66$ & $4.96 \pm 0.93$ & $<0.05$ & 0.01806 \\
\hline & FGB & $4.44 \pm 0.45$ & $4.82 \pm 0.70$ & $<0.05$ & 0.04980 \\
\hline & FGC & $4.31 \pm 0.38$ & $4.75 \pm 0.67$ & $<0.05$ & 0.01541 \\
\hline uPAR & PLAUR & $14.45 \pm 0.71$ & $13.96 \pm 0.57$ & $<0.05$ & 0.01965 \\
\hline Laminin 8 & LAMC1 & $9.23 \pm 0.82$ & $8.72 \pm 0.75$ & $<0.05$ & 0.04718 \\
\hline VCAM-1 & VCAM1 & $5.83 \pm 1.49$ & $5.64 \pm 1.17$ & - & 0.65764 \\
\hline
\end{tabular}

P-values denote PE vs. control groups. PE, pulmonary embolism; uPar, urokinase-type plasminogen activator receptor.

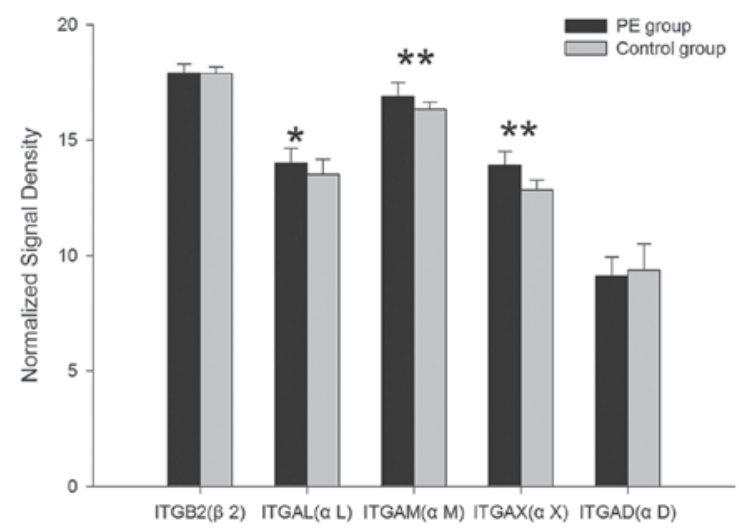

Figure 1. mRNA expression of genes associated with the subunits of $\beta 2$ integrins. ${ }^{*} \mathrm{P}<0.05 ;{ }^{* *} \mathrm{P}<0.01$. $\mathrm{PE}$, pulmonary embolism.

the following criteria: i) selective pulmonary arteriography showing a filling defect or blockage; ii) pulmonary ventilation perfusion scanning exhibiting single or multiple blood flow perfusion defects with normal or abnormal ventilation and mismatched ratio of ventilation/perfusion and iii) other clinical characteristics, including a typical manifestation of PE. Arterial

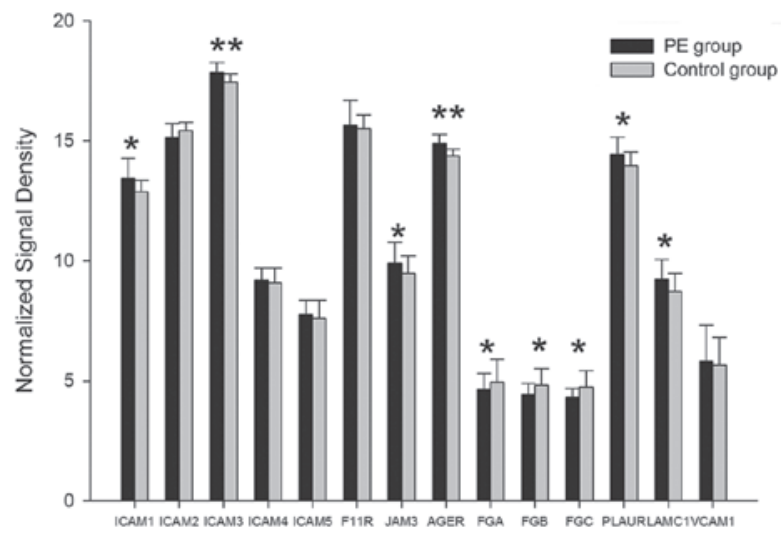

Figure 2. mRNA expression of genes associated with the ligand of $\beta 2$ integrins. ${ }^{*} \mathrm{P}<0.05 ;{ }^{* *} \mathrm{P}<0.01$. $\mathrm{PE}$, pulmonary embolism.

blood gas analysis, D-dimer test, ultrasound cardiogram and chest computerized tomography were used to support the diagnosis and exclude other cardiac and pulmonary disorders. A further 20 patients (11 males, 9 females; 44-91 years of age with a mean age of $72 \pm 14$ ) with ischemic heart disease admitted during the same period, without PE, DVT and other congenital 
Table III. mRNA expression of genes related to the chemokines.

\begin{tabular}{|c|c|c|c|c|c|}
\hline Chemokine & Gene & PE & Control & P-value & P-value \\
\hline SDF- $1 \alpha$ & CXCL12 & $5.76 \pm 0.43$ & $5.56 \pm 1.22$ & $<0.01$ & 0.00459 \\
\hline BLC & CXCL13 & $4.65 \pm 0.40$ & $5.01 \pm 0.69$ & - & 0.05251 \\
\hline SLC & CCL21 & $8.12 \pm 0.27$ & $8.11 \pm 0.50$ & - & 0.93293 \\
\hline RANTES & CCL5 & $16.37 \pm 0.63$ & $16.69 \pm 0.36$ & - & 0.06193 \\
\hline fMLP & FPR1 & $17.85 \pm 0.63$ & $17.71 \pm 0.62$ & - & 0.48139 \\
\hline IL-8 & IL8 & $11.27 \pm 1.86$ & $12.17 \pm 1.62$ & - & 0.11033 \\
\hline PAF & PAF1 & $11.96 \pm 0.54$ & $11.35 \pm 0.43$ & $<0.01$ & 0.00028 \\
\hline
\end{tabular}

P-values denote PE vs. control groups. PE, pulmonary embolism.

Table IV. mRNA expression of Rap1-related signals in inside-out activation.

\begin{tabular}{|c|c|c|c|c|c|}
\hline Protein & Gene & $\mathrm{PE}$ & Control & P-value & P-value \\
\hline \multirow[t]{2}{*}{$\operatorname{PLC} \gamma$} & PLCG1 & $10.23 \pm 0.96$ & $10.1 \pm 0.55$ & - & 0.48225 \\
\hline & PLCG2 & $15.36 \pm 0.44$ & $14.91 \pm 0.39$ & $<0.01$ & 0.00142 \\
\hline SLP-76 & $\mathrm{LCP} 2$ & $16.49 \pm 0.34$ & $16.16 \pm 0.26$ & $<0.01$ & 0.00142 \\
\hline ADAP & FYB & $16.68 \pm 0.35$ & $16.44 \pm 0.37$ & $<0.05$ & 0.04653 \\
\hline DalDAG-GEFI & RASGRP1 & $12.31 \pm 0.53$ & $12.39 \pm 0.38$ & $<0.05$ & 0.03376 \\
\hline SPA1 & SIPA1 & $16.23 \pm 0.46$ & $15.75 \pm 0.38$ & $<0.01$ & 0.00089 \\
\hline RIAM & APBB1IP & $15.83 \pm 0.44$ & $15.32 \pm 0.38$ & $<0.01$ & 0.00068 \\
\hline RAPL & RASSF5 & $14.16 \pm 0.95$ & $13.28 \pm 0.77$ & $<0.01$ & 0.00039 \\
\hline Mst1 & MST1 & $11.35 \pm 0.51$ & $11.55 \pm 0.49$ & - & 0.22278 \\
\hline
\end{tabular}

P-values denote PE vs. control groups. PE, pulmonary embolism Rap1, Ras-related protein 1.

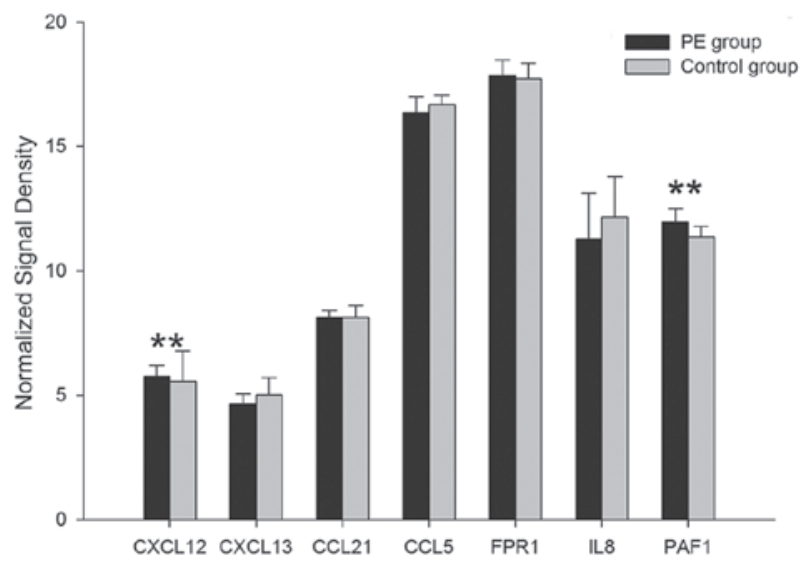

Figure 3. mRNA expression of genes associated with chemokines. ${ }^{* *} \mathrm{P}<0.01$, PE vs. control groups. PE, pulmonary embolism.

bleeding and thrombosis diseases, with comparative clinical presentation were enrolled in the control group. The study was approved by the Ethics Committee of Tongji University (Shanghai, China) and informed consent was obtained from all patients in accordance with the Declaration of Helsinki.

Total RNA isolation. A total of $5 \mathrm{ml}$ of peripheral blood samples anti-coagulated with EDTA were drawn from

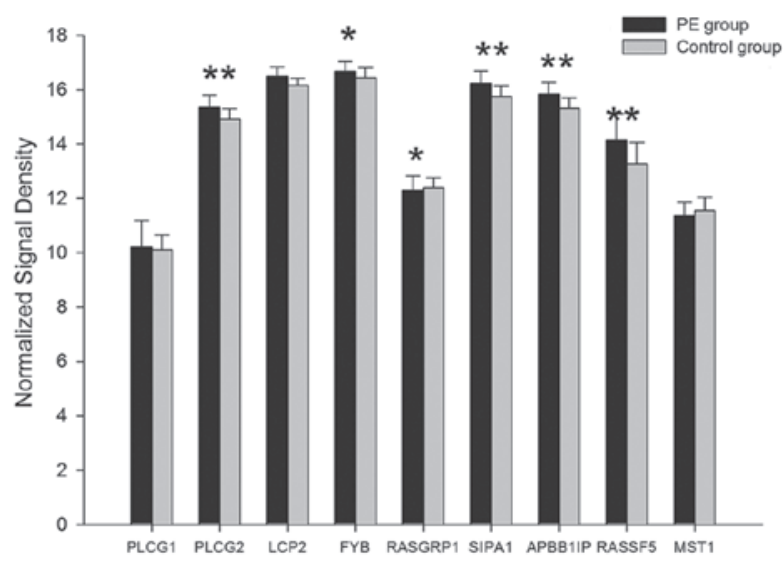

Figure 4. mRNA expression of Rap1 related signals in inside-out activation. ${ }^{*} \mathrm{P}<0.05 ;{ }^{* *} \mathrm{P}<0.01$, PE vs. control groups. PE, pulmonary embolism; Rap1, Ras-related protein 1 .

patients suspected of having PE and from those without PE, immediately following admission to the hospital. Leukocytes were obtained by density gradient centrifugation with Ficoll solution and the remaining red blood cells were destroyed by erythrocyte lysis buffer (Qiagen, Hilden, Germany). Total mononuclear cell RNA was extracted with TRIzol (Invitrogen Life Technologies, Carlsbad, CA, USA) and purified with 
Table V. mRNA expression of other proteins in inside-out activation.

\begin{tabular}{llcccc}
\hline Protein & Gene & PE & Control & P-value & P-value \\
\hline Talin & TLN1 & $13.28 \pm 1.18$ & $11.87 \pm 1.19$ & $<0.01$ & 0.00056 \\
& TLN2 & $6.166 \pm 0.43$ & $5.667 \pm 0.64$ & $<0.05$ & 0.01142 \\
Vinculin & VCL & $14.18 \pm 0.66$ & $13.32 \pm 0.85$ & $<0.01$ & 0.00109 \\
Dok1 & DOK1 & $12.17 \pm 0.4$ & $11.5 \pm 0.3$ & $<0.01$ & 0.00208 \\
$14-3-3 \zeta$ & YWHAZ & $15.67 \pm 0.41$ & $15.19 \pm 0.41$ & $<0.01$ & 0.00080 \\
Filamin A & FLNA & $16.16 \pm 0.74$ & $15.42 \pm 0.82$ & $<0.01$ & 0.00447 \\
Migfilin & FBLIM1 & $7.334 \pm 1.13$ & $7.206 \pm 1.3$ & $<0.05$ & 0.01134 \\
$\alpha$-actinin & ACTN1 & $16.62 \pm 0.61$ & $15.95 \pm 0.51$ & $<0.01$ & 0.00059 \\
Radixin & RDX & $10.81 \pm 0.41$ & $10.59 \pm 0.33$ & - & 0.07200 \\
Calpain & CAPN1 & $13.8 \pm 0.98$ & $12.62 \pm 0.96$ & $<0.01$ & 0.00044 \\
& CAPN3 & $12.45 \pm 0.48$ & $11.96 \pm 0.52$ & $<0.01$ & 0.00421 \\
& CAPN10 & $12.7 \pm 0.62$ & $12.42 \pm 0.46$ & $<0.05$ & 0.04066 \\
& CAPN11 & $6.494 \pm 1.13$ & $5.344 \pm 1.05$ & $<0.01$ & 0.00184 \\
& CAPN13 & $6.303 \pm 1.58$ & $5.59 \pm 1.13$ & $<0.05$ & 0.01328 \\
\hline
\end{tabular}

P-values denote PE vs. control groups. PE, pulmonary embolism.

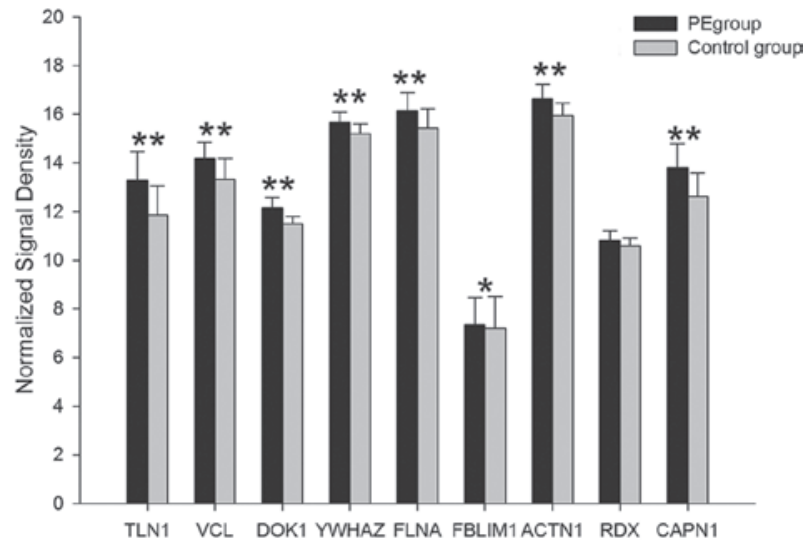

Figure 5. mRNA expression of other proteins in inside-out activation. ${ }^{*} \mathrm{P}<0.05 ;{ }^{* *} \mathrm{P}<0.01$, PE vs. control groups. $\mathrm{PE}$, pulmonary embolism.

RNeasy column (Qiagen), according to the manufacturer's instructions. The isolated total RNA was tested and quantified using a Nanodrop ND-1000 spectrophotometer (Thermo Fisher Scientific, Waltham, MA, USA).

Gene expression clip. Agilent G4112A Whole Human Genome Oligo Microarrays were purchased from Agilent Technologies Inc. (Santa Clara, CA, USA). The genes or transcripts included 314 negative control spots, 1,924 positive control spots and 359 blank spots. The functions of $>70 \%$ of the genes in the microarray have been previously identified. All patients were subjected to clip analysis.

Target preparation and microarray hybridization. The RNA samples of patients with confirmed diagnosis of PE and controls were labeled using the indirect labeling method. Briefly, $1 \mu \mathrm{g}$ of total RNA was reverse transcribed. Second strand cDNA was produced and purified, followed by in vitro transcription (IVT) with T7 RNA Polymerase. During IVT, the modified nucleotide, 5-(3-aminoallyl)-UTP (aaUTP) was incorporated into the cDNA. Subsequently, the fluorescent Cy3 was chemically coupled with the aaUTP, which contains a reactive primary amino group on the $\mathrm{C} 5$ position of uracil. The dye incorporation rate was assessed with a Nanodrop ND-1000 spectrophotometer and was found to be between 1.2 and $1.4 \mathrm{pmol} / \mu \mathrm{l}$. Hybridization was performed using the Agilent Oligonucleotide Microarray in situ Hybridization Plus kit (p/n 5,184-3,568), according to the manufacturer's instructions. Briefly, $750 \mathrm{ng}$ of $\mathrm{Cy} 3$-labeled sample cDNA was subjected to fragmentation $\left(30 \mathrm{~min}\right.$ at $\left.60^{\circ} \mathrm{C}\right)$ and hybridization on 44K Human Whole-Genome 60-mer oligo-chips (G4112F, Agilent Technologies) was performed in a rotary oven $\left(10 \mathrm{rpm}, 60^{\circ} \mathrm{C}, 17 \mathrm{~h}\right)$. Slides were disassembled and washed in solutions I and II, according to the manufacturer's instructions.

Reverse transcription-polymerase chain reaction (RT-PCR). Three differential genes were selected and their expression was confirmed by RT-PCR. Among the genes with differential expression, seven genes were randomly selected and the house keeping gene [glyceraldehyde 3-phosphate dehydrogenase (GAPDH)] was subjected to RT-PCR. The relative expression levels were indicated as the expression of the target genes normalized to the expression of GAPDH $\left(2^{-\Delta \Delta C t}\right)$. A melting curve and the $2^{-\Delta \Delta \mathrm{Ct}}$ method were used to compare the differences in the expression between the control and PE group. The results from RT-PCR were consistent with the microarray analysis.

Statistical analysis. Measurement data are expressed as the mean \pm SD. The Agilent Feature Extraction software was used to collect the original data from the microarray, followed by an analysis with a robust multichip average. The gene intensity data between the PE and control group were compared with a random variance model-corrected Student's t-test by SPSS 14.0 software packet (SPSS, Inc., Chicago, IL, USA). 
Table VI. mRNA expression of SFK and PKC in outside-in activation.

\begin{tabular}{|c|c|c|c|c|c|}
\hline SFK & Gene & $\mathrm{PE}$ & Control & P-value & P-value \\
\hline Hck & HCK & $16.49 \pm 0.73$ & $15.79 \pm 0.54$ & $<0.01$ & 0.00128 \\
\hline $\mathrm{Fgr}$ & FGR & $13.63 \pm 1.05$ & $12.55 \pm 0.68$ & $<0.01$ & 0.00044 \\
\hline Lyn & LYN & $17.47 \pm 0.52$ & $17.06 \pm 0.45$ & $<0.05$ & 0.01608 \\
\hline Lck & LCK & $15.72 \pm 0.56$ & $15.95 \pm 0.37$ & - & 0.34016 \\
\hline \multirow[t]{12}{*}{ PKC } & PRKCA & $7.127 \pm 0.67$ & $6.268 \pm 0.59$ & $<0.01$ & 0.00011 \\
\hline & PRKCB1 & $15.82 \pm 0.58$ & $15.36 \pm 0.38$ & $<0.01$ & 0.00511 \\
\hline & PRKCBP1 & $7.599 \pm 0.48$ & $6.902 \pm 0.39$ & $<0.01$ & 0.00473 \\
\hline & PRKCD & $16.44 \pm 0.67$ & $15.71 \pm 0.44$ & $<0.01$ & 0.00015 \\
\hline & PRKCDBP & $8.591 \pm 0.84$ & $8.226 \pm 0.68$ & $<0.01$ & 0.00653 \\
\hline & PRKCE & $6.308 \pm 0.41$ & $6.212 \pm 0.22$ & - & 0.3829 \\
\hline & PRKCG & $6.209 \pm 1.11$ & $6.179 \pm 1.16$ & - & 0.93199 \\
\hline & PRKCH & $8.351 \pm 0.5$ & $8.237 \pm 0.34$ & $<0.05$ & 0.01567 \\
\hline & PRKCI & $11.39 \pm 0.46$ & $11.51 \pm 0.3$ & - & 0.18020 \\
\hline & PRKCQ & $12.53 \pm 0.53$ & $12.69 \pm 0.35$ & - & 0.26876 \\
\hline & PRKCSH & $12.4 \pm 0.82$ & $11.55 \pm 0.69$ & $<0.01$ & 0.00111 \\
\hline & PRKCZ & $9.199 \pm 0.44$ & $9.379 \pm 0.41$ & - & 0.70588 \\
\hline
\end{tabular}

P-values denote PE vs. control groups. PE, pulmonary embolism; SFK, Src family kinase; PKC, protein kinase C.

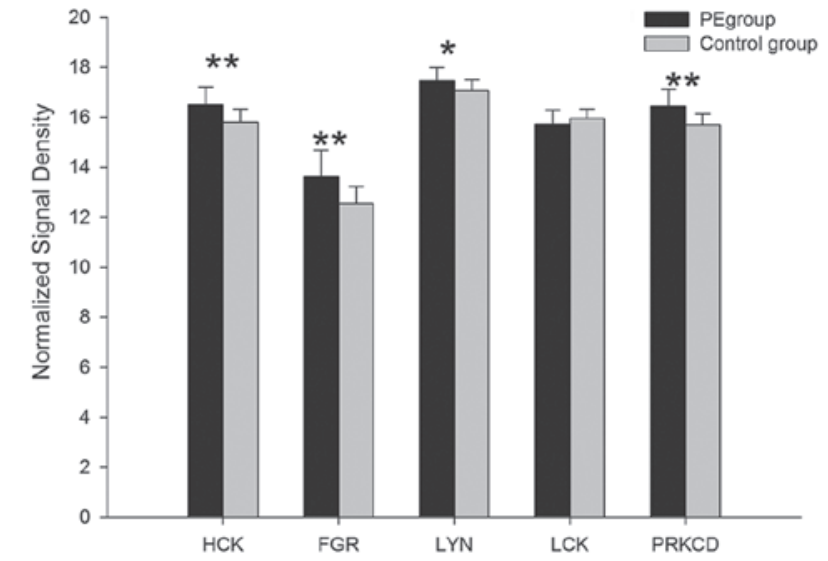

Figure 6. mRNA expression of SFK and PKC in outside-in activation. ${ }^{*} \mathrm{P}<0.05 ;{ }^{* *} \mathrm{P}<0.01$, PE vs. control groups. PE, pulmonary embolism; SFK, Src family kinase; PCK, protein kinase C.

Differentially expressed genes were identified from whole genomes. $\mathrm{P}<0.05$ was considered to indicate a statistically significant difference.

\section{Results}

A total of 80 associated mRNAs were detected (Tables I-VIII, Figs. 1-8). 14-3-3 $\zeta$ occurred in bilateral signal transduction pathways so was detected twice; once in inside-out activation, once in outside-in activation.

$m R N A$ expression of $\alpha / \beta$ integrins. Among the 5 subunit-associated mRNAs, the mRNA expression of ITGAL, ITGAM, ITGAX and ITGB2, which encode for the subunits of $\alpha \mathrm{L}$, $\alpha \mathrm{M}, \alpha \mathrm{X}, \beta 2$, were upregulated in the PE group and the differences, with the exception of ITGB2, exhibited statistical significance $(\mathrm{P}<0.05)$. Compared with the controls, the mRNA expression of ITGAD in the PE group was downregulated, but there was no significant difference $(\mathrm{P}>0.05)$ (Table I; Fig. 1).

mRNA expression of 12 ligands of $\beta 2$ integrins. Among the 14 associated mRNAs of 12 ligands, the gene expression of 10 ligands, ICAM-1, ICAM-3, ICAM-4, ICAM-5, JAM-1, JAM-3, RAGE, urokinase-type plasminogen activator receptor (UPAR), laminin 8 and VCAM-1, were upregulated in the PE group and the differences of ICAM-1, ICAM-3, JAM-3, AGER, PLAUR and LAMC1 exhibited statistical significance $(\mathrm{P}<0.05)$. Compared with the controls, the mRNA expression of ICAM-2 and fibrinogen were downregulated. The differences of FGA, FGB and FGG mRNA exhibited statistical significance $(\mathrm{P}<0.05)$; however, there was no significant difference for ICAM-2 (P>0.05) (Table II, Fig. 2).

mRNA expression of 7 chemokines for $\beta 2$ integrins. Among the 7 associated mRNAs of 7 chemokines for $\beta 2$ integrins, the gene expression of 6 chemokines, SDF-1 $\alpha$, SLC, RANTES, fMLP, IL-8 and PAF, were upregulated in the PE group and the differences of CXCL12 and PAF1 mRNA exhibited statistical significance $(\mathrm{P}<0.01)$. Compared with the controls, the mRNA expression of CXCL13 in the PE group was downregulated; however, this was not observed to be statistically significant (P>0.05) (Table III; Fig. 3).

mRNA expression of 33 signal transduction proteins. A total of 55 associated mRNAs of 33 signal transduction proteins which related to the $\beta 2$ integrins were detected:

i) Inside-out activation of the $\beta 2$ integrins. Among the 23 associated mRNAs of 17 inside-out signal proteins which related to $\beta 2$ integrins, the mRNA expression of 15 proteins, 
Table VII. mRNA expression of SYK-related proteins in outside-in activation.

\begin{tabular}{|c|c|c|c|c|c|}
\hline Name & Gene & $\mathrm{PE}$ & Control & P-value & P-value \\
\hline Syk & SYK & $15.70 \pm 0.49$ & $15.34 \pm 0.37$ & $<0.05$ & 0.01133 \\
\hline \multirow[t]{2}{*}{ Vav1/3 } & VAV1 & $15.93 \pm 0.45$ & $15.49 \pm 0.33$ & $<0.01$ & 0.00130 \\
\hline & VAV3 & $10.71 \pm 0.47$ & $10.14 \pm 0.43$ & $<0.01$ & 0.00176 \\
\hline \multirow[t]{2}{*}{$c-a b 1$} & ABL1 & $12.18 \pm 0.48$ & $11.85 \pm 0.41$ & $<0.05$ & 0.02491 \\
\hline & ABL2 & $8.336 \pm 0.56$ & $7.952 \pm 0.50$ & $<0.05$ & 0.02867 \\
\hline c-cb1 & CBL & $8.773 \pm 0.36$ & $7.921 \pm 0.40$ & $<0.01$ & 0.00066 \\
\hline FAK & PTK2 & $10.59 \pm 0.49$ & $10.08 \pm 0.48$ & $<0.01$ & 0.00632 \\
\hline
\end{tabular}

P-values denote PE vs. control groups. PE, pulmonary embolism; SYK, spleen tyrosine kinase.

Table VIII. $\beta 2$ mRNA expression of proteins which mediate cytoskeletal remodelling in outside-in activation.

\begin{tabular}{llcccc}
\hline Name & Gene & PE & Control & P-value & P-value \\
\hline $14-3-3 \zeta$ & YWHAZ & $15.67 \pm 0.41$ & $15.19 \pm 0.41$ & $<0.01$ & 0.0008 \\
Rac & RAC1 & $14.37 \pm 0.41$ & $13.72 \pm 0.30$ & $<0.01$ & 0.00104 \\
cdc42 & CDC42 & $13.80 \pm 0.41$ & $13.69 \pm 0.24$ & - & 0.56060 \\
RhoA & RHOA & $15.22 \pm 0.37$ & $14.86 \pm 0.22$ & $<0.01$ & 0.00579 \\
ROCK1/2 & ROCK1 & $13.11 \pm 0.53$ & $12.64 \pm 0.32$ & $<0.01$ & 0.00739 \\
& ROCK2 & $10.73 \pm 0.55$ & $10.27 \pm 0.47$ & $<0.05$ & 0.01867 \\
mDia & DIAPH1 & $14.76 \pm 0.35$ & $14.31 \pm 0.35$ & $<0.01$ & 0.00107 \\
& DIAPH2 & $12.07 \pm 0.47$ & $11.78 \pm 0.50$ & $<0.05$ & 0.03168 \\
& DIAPH3 & $5.382 \pm 0.94$ & $5.332 \pm 0.98$ & - & 0.29674 \\
\hline
\end{tabular}

P-values denote PE vs. control groups. PE, pulmonary embolism.

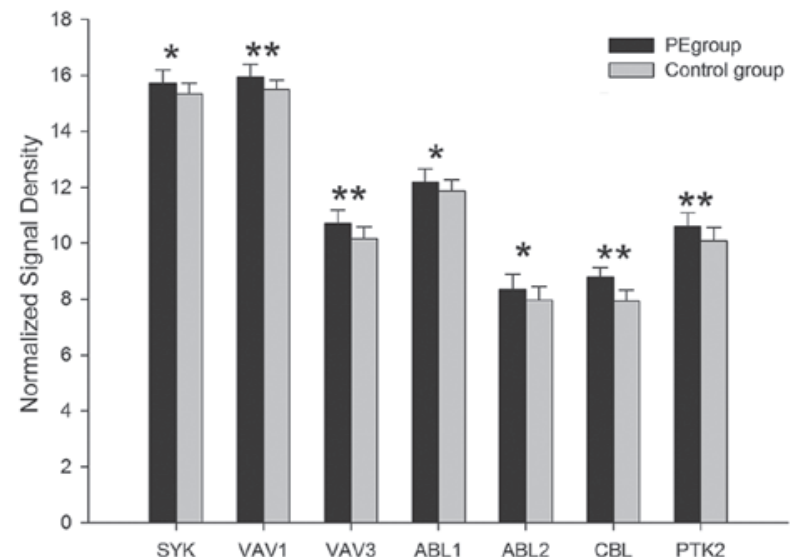

Figure 7. mRNA expression of SYK related proteins in outside-in activation. ${ }^{*} \mathrm{P}<0.05 ;{ }^{* *} \mathrm{P}<0.01, \mathrm{PE}$ vs. control groups. PE, pulmonary embolism; SYK, spleen tyrosine kinase

PLC $\gamma$, SPA1, SLP-76, ADAP, RIAM, RAPL, Talin, Vinculin, Dok1, 14-3-3̧, Filamin A, Migfilin, $\alpha$-actinin, Radixin and Calpain, were upregulated in the PE group. The differences in PLCG2, SIPA1, LCP2, FYB, APBB1IP, RASSF5, TLN1, TLN2, VCL, DOK1, YWHAZ, FLNA, FBLIM1, ACTN1 and CAPN1 mRNA were observed to be statistically significant $(\mathrm{P}<0.05)$. Compared with the controls, the mRNA expression of DalDAG-GEFI and Mst1 in the PE group were

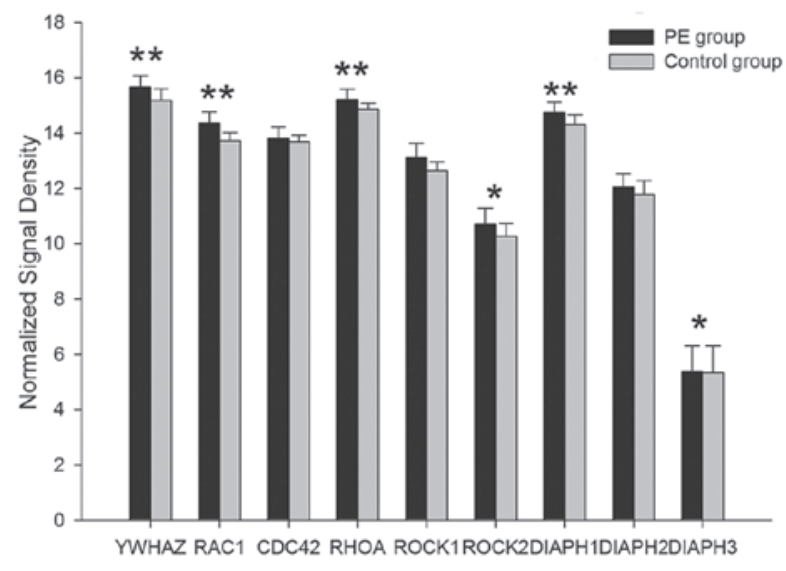

Figure 8. mRNA expression of proteins which mediate cytoskeletal remodelling in outside-in activation. ${ }^{*} \mathrm{P}<0.05,{ }^{* *} \mathrm{P}<0.01, \mathrm{PE}$ vs. control groups. $\mathrm{PE}$, pulmonary embolism.

downregulated. The difference of DalDAG-GEFI encoded by RASGRP1 mRNA exhibited statistical significance $(\mathrm{P}<0.05)$, however there was no significant difference for Mst1 mRNA ( $\mathrm{P}>0.05)$ (Tables IV and V; Figs. 4 and 5).

ii) Outside-in activation of the $\beta 2$ integrins. Among the 32 associated mRNAs of 16 outside-in signal proteins which are related to $\beta 2$ integrins, the gene expression of 14 proteins, 
Table IX. The expression, ligands and functions of $\beta 2$ integrins.

\begin{tabular}{|c|c|c|c|c|c|}
\hline Integrin & Other names & Expression & Ligands & Function & Ref. \\
\hline$\alpha \mathrm{L} \beta 2$ & $\begin{array}{l}\text { CD11a/CD18 } \\
\text { LFA-1 }\end{array}$ & All leukocytes & $\begin{array}{l}\text { ICAM-1, ICAM-2, } \\
\text { ICAM-3, ICAM-4, } \\
\text { ICAM-5, JAM-1 }\end{array}$ & $\begin{array}{l}\text { Mediates leukocyte infiltration; } \\
\text { involved in immune } \\
\text { synapse formation }\end{array}$ & $\begin{array}{c}8 \\
9,10\end{array}$ \\
\hline$\alpha \mathrm{M} \beta 2$ & $\begin{array}{l}\text { CD11b/CD18 } \\
\text { Mac-1 }\end{array}$ & $\begin{array}{l}\text { Monocytes, neutrophils } \\
\text { macrophages, NK cells } \\
\text { and } \gamma \delta \text { T-cells }\end{array}$ & $\begin{array}{l}\text { ICAM-1, ICAM-2, } \\
\text { ICAM-4, JAM-3, } \\
\text { RAGE, laminin 8, } \\
\text { fibrinogen and more }\end{array}$ & $\begin{array}{l}\text { Mediates leukocyte phagocytosis; } \\
\text { mediates leukocyte adhesion; } \\
\text { involved in immune tolerance }\end{array}$ & $\begin{array}{c}11 \\
12,13\end{array}$ \\
\hline$\alpha \mathrm{X} \beta 2$ & $\begin{array}{l}\text { CD11c/CD18 } \\
\text { p } 150,95\end{array}$ & $\begin{array}{l}\text { Monocytes, NK cells, } \\
\text { macrophages } \\
\text { and dendritic cells }\end{array}$ & $\begin{array}{l}\text { ICAM-1, ICAM-4, } \\
\text { fibrinogen, LPS } \\
\text { and more }\end{array}$ & $\begin{array}{l}\text { Mediates the adhesion between } \\
\text { monocytes and endotheliocytes }\end{array}$ & \\
\hline$\alpha \mathrm{D} \beta 2$ & CD11d/CD18 & $\begin{array}{l}\text { Macrophages } \\
\text { and eosinophils }\end{array}$ & $\begin{array}{l}\text { ICAM-3, VCAM-1 } \\
\text { and more }\end{array}$ & $\begin{array}{l}\text { Involved in the phagocytosis of } \\
\text { senescent erythrocyte; } \\
\text { promotes eosinophil infiltration }\end{array}$ & 14 \\
\hline
\end{tabular}

Hck, Lyn, PKC, Syk, Vav1/3, c-ab1, c-cb1, FAK, 14-3-3६, Rac, cdc42, RhoA, ROCK1/2 and mDia, were upregulated in the PE group and the differences, with the exception of cdc42, exhibited statistical significance $(\mathrm{P}<0.05)$. Compared with the controls, the mRNA expression of Fgr and Lck1 in the PE group was downregulated. The difference of Fgr encoded by FGR mRNA exhibited statistical significance $(\mathrm{P}<0.01)$, however, alterations in LCK mRNA were not observed to be statistically significant ( $\mathrm{P}>0.05$ ) (Tables VI-VIII; Figs. 6-8).

\section{Discussion}

Neutrophils and monocytes are indispensable parts of innate immunity. During an inflammatory response, neutrophils and monocytes destroy invading pathogens by phagocytosis. In 2009 , it was observed (3) that the associated mRNA expression of neutrophils and monocytes in PE patients were upregulated significantly $(\mathrm{P}<0.01) . \beta 2$ integrins are the primary adhesion molecules for leukocyte adhesion in the inflammatory response and are also significant in the phagocytosis of neutrophils and monocytes.

The leukocyte-restricted $\beta 2$ integrins include four members: $\alpha \mathrm{L} \beta 2, \alpha \mathrm{M} \beta 2, \alpha \mathrm{X} \beta 2$ and $\alpha \mathrm{D} \beta 2$ (Table IX) (7-13). The exudation of neutrophils or monocytes includes margination, rolling, stable adhesion and transendothelial migration. The stable adhesion occurs mainly through the interaction between $\alpha \mathrm{L} \beta 2$ and $\alpha \mathrm{M} \beta 2$ and their ligands on the surface of cytomembrane. When inflammation occurs, the expression of chemokines is upregulated. The inside-out signal transduction is activated following chemokine binding with the receptors on the surface of neutrophils and monocytes. Following G-protein signaling, activated phospholipase C (PLC) mediates the activation of DalDAG-GEFI. Ras-related protein 1 (Rap1) is a small GTPase, which is a key protein in the inside-out signaling. SPA1 may convert activated Rap1 to inactive Rap1. DalDAG-GEFI may convert inactive Rap1 to activated Rap1 and subsequently activate the downstream effectors of Rap1, including RIAM, RAPL and Mst1. The effectors may regulate the ligand-binding affinity of the $\beta 2$ integrins via cytoskeletal proteins which bind to the integrin $\beta$ cytoplasmic tail, including Talin, Dok1, 14-3-3६, Filamin A, Migfilin and Radixin, eventually improving the affinity of the $\beta 2$ integrins and promoting the adhesion of leukocytes.

The results of the current study showed that the mRNA expression of associated chemokines were upregulated in the PE group, compared with the controls. The differences of SDF- $1 \alpha$ and PAF1 mRNA were statistically significant $(\mathrm{P}<0.01)$. Among the 23 associated mRNAs of the 17 inside-out signal proteins which related to the $\beta 2$ integrins, the mRNA expression of 15 proteins was upregulated significantly in the $\mathrm{PE}$ group $(\mathrm{P}<0.05)$. Among the 5 subunit-associated mRNAs, the mRNA expression of ITGAL, ITGAM, ITGAX and ITGB2, which encode for the subunits of $\alpha \mathrm{L}, \alpha \mathrm{M}, \alpha \mathrm{X}$ and $\beta 2$, were upregulated in the PE group and the differences, with the exception of ITGB2, were observed to be statistically significant $(\mathrm{P}<0.05)$.

The current study suggested that the inside-out signal transduction pathway of the $\beta 2$ integrins was activated in the neutrophils and monocytes of PE patients. Given the overexpression of activated Rap1, the expression of DalDAG-GEFI was downregulated as the result of negative feedback. Filamin may compete with Talin to bind the integrin tail (6). As a negative regulator of integrin activation, the mRNA expression of Filamin A was upregulated significantly for the overexpression of activated Rap1. The associated chemokines SDF- $1 \alpha$, RANTES, fMLP, IL- 8 and PAF promote the migration of leukocytes through the endothelium (7). The differences of SDF-1 $\alpha$ and PAF1 mRNA were statistically significant $(\mathrm{P}<0.01)$, suggesting that the exudation of neutrophils and monocytes is enhanced. The gene expression of Talin, Dok1, 14-3-3ร, Filamin A, Migfilin and Skelemin were upregulated significantly $(\mathrm{P}<0.01)$. It suggested that the $\beta 2$ integrins were activated, neutrophils and monocytes had high-affinity to the endothelium and the adhesive attraction was enhanced.

Neutrophils and monocytes associated with the phagocyte. The phagocytosis of neutrophils or monocytes includes recognition, adhesion, ingestion and degradation. Depending on the Fc receptor and $\mathrm{C} 3 \mathrm{~b}$ receptor (C3bi or $\alpha \mathrm{M} \beta 2)$, neutrophils and monocytes may recognize and adhere the bacteria which are 
coated with the antibody or complement and ingest the phagosome via the cytoskeletal reorganization.

Phagocytosis mediated by $\beta 2$ integrins primarily depends on the outside-in signal transduction pathway of $\alpha \mathrm{M} \beta 2$. When the $\beta 2$ integrins of neutrophils and monocytes bind with the ligand, the outside-in signaling events are initiated. Non-receptor tyrosine kinase and the PTK (receptor tyrosine kinase), including spleen tyrosine kinase (SYK) and FAK, are activated through the phosphorylation of Src family kinase (SFKs), including Hck, Fgr and Lyn. SFKs and SYK are required for the regulation of adhesion, spread and cytoskeletal reorganization in neutrophils and monocytes. Under the effect of Vav (a Rac GEF), cross-linking of the $\beta 2$ integrins may induce the activation of RhoA. The activated RhoA regulates the cytoskeletal structures and promotes the phagocytosis of leukocytes. Protein kinase $\mathrm{C}$ (PKC) promotes the phagocytosis of leukocytes. uPAR is required for the adhesion reaction of endothelial cells with monocytes (14).

The results of the current study showed that among the 14 associated mRNAs of 12 ligands, the gene expression of 10 ligands was upregulated in the PE group. The differences of ICAM-1, ICAM-3, JAM-3, AGER, PLAUR and LAMC1 was statistically significant $(\mathrm{P}<0.05)$. Compared with the controls, the mRNA expression of ICAM-2 and Fibrinogen were downregulated. There were significant differences of FGA, FGB and FGG mRNA expression $(\mathrm{P}<0.05)$. Among the 32 associated mRNAs of 16 outside-in signaling proteins which related to the $\beta 2$ integrins, the mRNA expression of 14 proteins were upregulated in the PE group and the differences, with the exception of cdc42, were statistically significant $(\mathrm{P}<0.05)$. Compared with the controls, the mRNA expression of Fgr and Lck1 in the PE group were downregulated. The difference of FGR mRNA was statistically significant $(\mathrm{P}<0.01)$.

It is hypothesized that the outside-in signal transduction pathway of $\beta 2$ integrins was activated in the neutrophils and monocytes of PE patients. The mRNA expression of Hck, PKC, FAK and RhoA were upregulated significantly $(\mathrm{P}<0.01)$. The mRNA expression of Lyn and SYK were markedly upregulated $(\mathrm{P}<0.05)$. These observations suggest that the phagocytosis of neutrophils and monocytes is enhanced. Fgr negatively regulates the migration and adhesion via the signal transduction pathway of the $\beta 2$ integrins. Compared with the controls, there was no negative feedback rise in the mRNA expression of $\mathrm{Fgr}$, instead, it was downregulated significantly $(\mathrm{P}<0.01)$. Although the $\beta 2$ integrins of leukocytes in PE patients were activated, the associated mRNA expression of Fgr is hypothesized to be inhibited.
The analysis of mRNA differential expression in leukocyte $\beta 2$ integrins signal transduction suggested that the bilateral signal transduction pathways of $\beta 2$ integrins were activated. The innate immune response in PE patients was enhanced. From the perspective of genomics, it is indicated that the occurrence of PE was apparently associated with the activation of $\beta 2$ integrins in neutrophils and monocytes. The occurrence of $\mathrm{PE}$ and the inflammatory reaction are closely related.

\section{References}

1. Smeeth L, Cook C, Thomas S, Hall AJ, Hubbard R and Vallance P. Risk of deep vein thrombosis and pulmonary embolism after acute infection in a community setting. Lancet 367: 1075-1079, 2006.

2. Haoming S, Lemin $\mathrm{W}$, Zhu G, et al: $\mathrm{T}$ cell mediated immune deficiency or compromise in patients with CTEPH. Am J Respir Crit Care Med 183: 417-418, 2011.

3. Gong Z, Liang AB, Wang LM, et al: The expression and significance of immunity associated genes mRNA in patients with pulmonary embolism. Zhonghua Nei Ke Za Zhi 48: 666-669, 2009 (In Chinese).

4. Plow EF, Haas TA, Zhang L, Loftus J and Smith JW: Ligand binding to integrins. J Biol Chem 275: 21785-21788, 2000.

5. Xie Y, Duan Q, Wang L, Gong Z, Wang Q, Song H and Wang H: Genomic characteristics of adhesion molecules in patients with symptomatic pulmonary embolism. Mol Med Rep 6: 585-590, 2012.

6. Kiema T, Lad Y, Jiang P, et al: The molecular basis of filamin binding to integrins and competition with talin. Mol Cell 21: 337-347, 2006.

7. Schenkel AR, Mamdouh Z and Muller WA: Locomotion of monocytes on endothelium is a critical step during extravasation. Nat Immunol 5: 393-400, 2004.

8. Iezzi G, Karjalainen K and Lanzavecchia A: The duration of antigenic stimulation determines the fate of naive and effector T cells. Immunity 8: 89-95, 1998.

9. Perez OD, Mitchell D, Jager GC, et al: Leukocyte functional antigen 1 lowers $\mathrm{T}$ cell activation thresholds and signaling through cytohesin-1 and Jun-activating binding protein 1 . Nat Immunol 4: 1083-1092, 2003.

10. Ehlers MR: CR3: a general purpose adhesion-recognition receptor essential for innate immunity. Microbes Infect 2: 289-294, 2000.

11. Varga G, Balkow S, Wild MK, et al: Active MAC-1 (CD11b/CD18) on DCs inhibits full T-cell activation. Blood 109: 661-669, 2007.

12. Han C, Jin J, Xu S, Liu H, Li N and Cao X: Integrin CD11b negatively regulates TLR-triggered inflammatory responses by activating Syk and promoting degradation of MyD88 and TRIF via Cbl-b. Nat Immunol 11: 734-742, 2010.

13. Danilenko DM, Rossitto PV, Van der Vieren M, Le Trong H, McDonough SP, Affolter VK and Moore PF: A novel canine leukointegrin, alpha $\mathrm{d}$ beta 2 , is expressed by specific macrophage subpopulations in tissue and a minor $\mathrm{CD}^{+}$lymphocyte subpopulation in peripheral blood. J Immunol 155: 35-44, 1995.

14. Andreas EM: Urokinase receptor surface expression regulates monocyte adhesion in acute myocardial infarction. Blood 100: 3611-3617, 2002. 